\title{
Case finding of early pregnancies at risk of preeclampsia using maternal blood leptin/ceramide ratio: multi-omics discovery and validation from a longitudinal study
}

\author{
Qianyang Huang ${ }^{1}$, Shiying $\mathrm{Hao}^{2}$, Jin $\mathrm{You}^{3}$, Xiaoming Yao ${ }^{1},{\mathrm{Zhen} \mathrm{Li}^{2} \text {, James Schilling }}^{1}$, \\ Zhen $\mathrm{Li}^{4}$, Sheeno Thyparambil ${ }^{1}$, Wei-Li Liao ${ }^{1}$, Xin Zhou ${ }^{5}$, Lihong $\mathrm{Mo}^{6}$, Subhashini \\ Ladella $^{6}$, David Fan ${ }^{7}$, John Whitin ${ }^{2}$, Harvey Cohen ${ }^{2}$, Doff McElhinney ${ }^{2}$, Ronald Wong ${ }^{2}$, \\ Gary Shaw ${ }^{2}$, David Stevenson ${ }^{8}$, Karl Sylvester ${ }^{2}$, and Xuefeng Ling ${ }^{2}$ \\ ${ }^{1} \mathrm{mProbe}$, Inc. \\ ${ }^{2}$ Stanford University School of Medicine \\ ${ }^{3}$ University of California Riverside \\ ${ }^{4}$ Southeast University \\ ${ }^{5}$ Pingjin Hospital Heart Center \\ ${ }^{6}$ University of California San Francisco Fresno Center for Medical Education and Research \\ ${ }^{7}$ University of California Santa Barbara \\ ${ }^{8}$ Lucile Salter Packard Children's Hospital at Stanford
}

January 20, 2021

\begin{abstract}
Objective To evaluate whether longitudinal measurements of serological adipokines and sphingolipids can predict preeclampsia early in gestation. Design Retrospective multi-omics discovery and longitudinal validation. Setting Maternity units in two US hospitals. Methods A multi-omics approach integrating genomic and lipidomic discoveries was employed to identify leptin (Lep) and ceramide (Cer) as novel PE early gestational biomarkers. The levels of placental growth factor (PlGF), soluble fms-like tyrosine kinase (sFlt-1), Lep, and Cer in maternal sera were then determined by enzyme-linked immunosorbent and liquid chromatography-tandem mass spectrometric assays. Main outcome measures Interval from positive prediction to confirmative diagnosis. Results Genomic meta-analysis compiled six PE placental cohorts with 78 PE and 95 non-PE control placentas. The Testing Cohort included sera from 7 non-PE and 8 PE women collected at confirmatory diagnosis. The Validation Cohort included sera from 20 non-PE and 20 PE women collected longitudinally through gestation. Our findings revealed a marked elevation of maternal serum Leptin/Ceramide (d18:1/25:0) ratio from early gestation (a median of 23 weeks) when comparing later PE-complicated with uncomplicated pregnancies. The maternal Lep/Cer (d18:1/25:0) ratio significantly outperformed the established sFlt-1/PlGF ratio in predicting PE for sensitivity ( $85 \%$ vs. $40 \%$ ), positive predictive value ( $89 \%$ vs. $42 \%$ ), and AUC (0.92 vs. 0.52) from 5 to 25 weeks of gestation. Conclusions Non-invasive longitudinal assessment by serological evaluation of Lep/Cer (d18:1/25:0) ratio can case find early pregnancies at risk of preeclampsia, outperforming sFlt-1/PlGF ratio test. Tweetable abstract Non-invasive longitudinal assessment by serological evaluation of Lep and Cer ratio can predict preeclampsia early in gestation.
\end{abstract}

Case finding of early pregnancies at risk of preeclampsia using maternal blood leptin/ceramide ratio: multi-omics discovery and validation from a longitudinal study

Running title: Maternal leptin and ceramide predict preeclampsia 
Qianyang Huang ${ }^{1 \#}$, Shiying $\mathrm{Hao}^{2,3 \#}$, Jin You ${ }^{4 \#}$, Xiaoming Yao ${ }^{1 \#}$, Zhen Li $^{5}$, James Schilling ${ }^{1,6}$, Zhen $\mathrm{Li}^{6,7}$, Sheeno Thyparambil ${ }^{1}$, Wei-li Liao ${ }^{1}$, Xin Zhou ${ }^{8}$, Lihong Mo ${ }^{9}$, Subhashini Ladella ${ }^{9}$, David Fan ${ }^{10}$, John C. Whitin ${ }^{11}$, Harvey J. Cohen ${ }^{11}$, Doff B. McElhinney ${ }^{2,3}$, Ronald J. Wong ${ }^{11}$, Gary M. Shaw ${ }^{11}$, David K. Stevenson $^{11}$, Karl G. Sylvester ${ }^{5}$, Xuefeng B. Ling ${ }^{3,5^{*}}$

${ }^{1}$ mProbe Inc., Mountain View, CA, United States

${ }^{2}$ Department of Cardiothoracic Surgery, Stanford University School of Medicine, Stanford, CA, United States

${ }^{3}$ Clinical and Translational Research Program, Betty Irene Moore Children's Heart Center, Lucile Packard Children's Hospital, Palo Alto, CA, United States

${ }^{4}$ Department of Bioengineering, University of California Riverside, Riverside, CA, United States

${ }^{5}$ Department of Surgery, Stanford University School of Medicine, Stanford, CA, United States

${ }^{6}$ Binhai Industrial Technology Research Institute, Zhejiang University, Tianjin, China

${ }^{7}$ School of Electrical Engineering, Southeast University, Nanjing, China

${ }^{8}$ Department of Cardiology, Tianjin Medical University General Hospital, Tianjin, China

${ }^{9}$ Department of Obstetrics and Gynecology, University of California San Francisco-Fresno, Fresno, CA, United States

${ }^{10}$ Department of Statistics and Applied Probability, University of California Santa Barbara, Santa Barbara, CA, United States

${ }^{11}$ Department of Pediatrics, Stanford University School of Medicine, Stanford, CA, United States

\#Co-first authors

* Corresponding author: Stanford University School of Medicine, Stanford, CA, 94305, USA; Tel: 650-4279198; Fax: (650)-723-1154; Email Address: bxling@stanford.edu

\section{Abstract}

\section{Objective}

To evaluate whether longitudinal measurements of serological adipokines and sphingolipids can predict preeclampsia early in gestation.

\section{Design}

Retrospective multi-omics discovery and longitudinal validation.

\section{Setting}

Maternity units in two US hospitals.

\section{Methods}

A multi-omics approach integrating genomic and lipidomic discoveries was employed to identify leptin (Lep) and ceramide (Cer) as novel PE early gestational biomarkers. The levels of placental growth factor (PlGF), soluble fms-like tyrosine kinase (sFlt-1), Lep, and Cer in maternal sera were then determined by enzymelinked immunosorbent and liquid chromatography-tandem mass spectrometric assays.

Main outcome measures

Interval from positive prediction to confirmative diagnosis.

\section{Results}


Genomic meta-analysis compiled six PE placental cohorts with 78 PE and 95 non-PE control placentas. The Testing Cohort included sera from 7 non-PE and 8 PE women collected at confirmatory diagnosis. The Validation Cohort included sera from 20 non-PE and 20 PE women collected longitudinally through gestation. Our findings revealed a marked elevation of maternal serum Leptin/Ceramide (d18:1/25:0) ratio from early gestation (a median of 23 weeks) when comparing later PE-complicated with uncomplicated pregnancies. The maternal Lep/Cer (d18:1/25:0) ratio significantly outperformed the established sFlt-1/PlGF ratio in predicting $\mathrm{PE}$ for sensitivity ( $85 \%$ vs. $40 \%$ ), positive predictive value ( $89 \%$ vs. $42 \%$ ), and AUC (0.92 vs. 0.52 ) from 5 to 25 weeks of gestation.

\section{Conclusions}

Non-invasive longitudinal assessment by serological evaluation of Lep/Cer (d18:1/25:0) ratio can case find early pregnancies at risk of preeclampsia, outperforming sFlt-1/PlGF ratio test.

\section{Tweetable abstract}

Non-invasive longitudinal assessment by serological evaluation of Lep and Cer ratio can predict preeclampsia early in gestation.

Keyword : Preeclampsia Prediction; Early Gestation; Leptin; Ceramide; Maternal Serum

\section{List of Abbreviation}

Acetyl-CoA carboxylase: ACC

Area Under the Curve: AUC

AMP-activated protein kinase: AMPKK/AMPK

Body Mass Index: BMI

Ceramide: Cer

Confidence Interval: CI

Dihydroceramide: DHCer

Enzyme-Linked Immunosorbent Assay: ELISA

Gene Expression Omnibus: GEO

Leptin: Lep

Liquid Chromatography-Tandem Mass Spectrometry: LC/MS/MS

Negative Predictive Value: NPV

Placental Growth Factor: PlGF

Positive Predictive Value: PPV

Preeclampsia: PE

Soluble fms-Like Tyrosine Kinase: sFlt-1

Wk: Week

\section{INTRODUCTION}

Preeclampsia (PE) is a disorder of the placental vasculature, affecting $5 \%$ to $8 \%$ of all pregnancies worldwide. It still remains a leading cause of maternal and fetal mortality, accounting for $42 \%$ of all maternal deaths and $15 \%$ of preterm deliveries . It is characterized by diffused endothelial dysfunction, increased peripheral vascular resistance, hypertension, proteinuria, and dysregulated coagulation. The pathogenesis of PE is complex as it progresses from asymptomatic stage in the first trimester to a symptomatic stage late in 
gestation. Although its etiologies remain largely unknown, mounting evidence has revealed that placental dysfunction is integral to the development of PE . Pathophysiological perturbations of placental development cause incomplete remodeling of the uterine spiral arteries and poor invasion of trophoblasts into placental cells, which induces persistent placental oxidative stress and hypoxia, such as PE . From a clinical perspective, early prediction of preeclampsia (i.e., within the first 16 weeks of gestion) is of critical importance as it would allow for early treatment of high-risk women, which has been proposed to reduce the occurrence of preeclampsia. Gestational interventions such as steroids to accelerate fetal lung maturity, magnesium for seizure prophylaxis, aspirin treatment, and antihypertensive therapy are effective in reducing both maternal and fetal mortality in populations with high risks of developing PE .

However, the early prediction of PE remains challenging. Traditional risk factors such as a prior history of $\mathrm{PE}$, first pregnancy, multiple gestation, and obesity have insufficient sensitivity and specificity (less than $60 \%)$ for the prediction of PE . An imbalance of angiogenic and anti-angiogenic factors during pregnancy was found to disrupt the developmental homeostasis of the placenta . Two placental-derived factors, angiogenic soluble fms-like tyrosine kinase (sFlt-1) and anti-angiogenic placental growth factor (PlGF), were associated with the pathophysiology of PE . A multicenter trial demonstrated that the sFlt-1/PlGF ratio in maternal sera significantly differentiates pregnant PE from normal pregnant women after 24 wks' of gestation . Later studies discovered that this ratio had limited value in predicting the development of PE when examined during the first or early second trimesters. Thus, there is an unmet need to identify sensitive and specific markers to predict PE early in gestation.

Previous studies have suggested that PE is a pregnancy complication that is associated with changes of multiple systems and encompasses genetic, proteomic, and metabolic factors . Recent multi-omics studies identified a number of molecular-level candidates associated with PE . One of these candidates is leptin (Lep), a secreted adipokine that affects the central regulation of energy homeostasis, neuroendocrine function, and cytoplasmic metabolism . Lep can be expressed by both adipose and non-adipose tissues, which, during pregnancy, not only mediates the gestational energy homeostasis, but also modulates various physiological events, such as implantation, placentation, and immune adaption, that are essential for fetal development - Our previous findings have demonstrated elevations of Lep in early gestation in PE patients . Other have also reported that sphingolipid metabolism, particularly via ceramide (Cer), acts downstream to the anorectic actions of central Lep, and played an important role in Lep-induced hypothalamic control of feeding . Furthermore, our recent findings have also illustrated the regulatory role of Cer as a metabolic messenger for the homeostatic development of normal pregnancy along gestation, and placental changes in cytoplasmic amount of Cer in trophoblast cells have been shown to be implicated in the pathogenesis of $\mathrm{PE}$.

In this study, we employed a multi-omics approach to identify Lep and Cer as potential biomarker candidates for risk of impending PE. This initial omics-based discovery led to the generation of our hypothesis that the gestational profiles of Lep and Cer differ in maternal serum from women without PE compared with those with PE. We further hypothesized that the ratio of Lep and Cer can serve as a serological marker capable of predicting impending PE early in gestation. We therefore characterized the serological profiles of circulating Lep and Cer longitudinally and investigated their potential utility in predicting impending PE early in pregnancy and biological insights.

\section{MATERIALS and METHODS}

\section{Study Design}

The overall sample allocation, hypothesis generation, biomarker discovery, independent validation, and panel construction workflows are illustrated in Fig. 1. Our study was conducted in three phases: (1) the discovery phase, which included both in-silico expression analysis of PE and non-PE placentas and comprehensive literature mining to generated the hypothesis that Lep and Cer might be implicated in PE pathophysiology as potential biomarkers; (2) the testing phase, which measured the levels of Lep and Cer in a case-control cohort of PE and non-PE maternal sera sampled at confirmative diagnosis; (3) the validation phase, which determined the levels of Lep and Cer in an independent longitudinal cohort of PE and non-PE maternal sera 
sampled at different gestational ages.

\section{Meta-analysis of placental gene expression}

Six PE placenta expression studies from Gene Expression Omnibus (GEO) datasets were combined and subjected to multiplex analysis with the method as we previously developed. We calculated the meta-fold change of each gene across all studies. For gene expression measurements, this corresponds to combining fold-changes across studies to identify a meta-fold-change that is an amalgamation of the constituent studies. The meta p-values were obtained by Fisher's method. Significant genes were selected if they were measured in five or more studies and the meta effect $\mathrm{p}$ value was less than $4.5 \times 10^{-5}$. This effort identified Lep as the leading protein biomarker candidate. We also compared the expression of Lep transcript between non-PE and $\mathrm{PE}$ women in individual datasets.

\section{Study population and blood collection}

Two independent cohorts of pregnant women were assembled for this study. The testing cohort included sera from women between 25 and 31 wks' of gestation with or without PE collected by ProMedDX Inc. (Norton, MA). Each woman had one sample collected. All PE samples were collected at the time of confirmative diagnosis. The validation cohort included sera from women who participated in a longitudinal study sponsored by the March of Dimes Prematurity Center at Stanford University between November 2012 and May 2016. Each woman had multiple sera collected from 5 to 29 wks' of gestation prior to PE diagnosis. Study was approved by ethics committees at Stanford University with an IRB\#21956 starting from Sep. 30 2011. Written informed consent was obtained from all participants.

The diagnosis of PE was made according to the American College of Obstetricians and Gynecologists criteria as follows: a persistent systolic blood pressure [?]140 $\mathrm{mmHg}$, or a diastolic blood pressure [?] $90 \mathrm{mmHg}$ after 20 wks' of gestation in a woman having a previous normal blood pressure in conjunction with one or more of the following: new-onset proteinuria, new-onset thrombocytopenia, impaired liver function, renal insufficiency, pulmonary edema, or visual or cerebral disturbances in the absence of proteinuria. Early-onset $\mathrm{PE}$ was defined as PE that develops before 34 wks' of gestation, whereas the late-onset PE develops at or after 34 wks' of gestation.

\section{Enzyme-linked immunosorbent assay (ELISA)}

Serum concentrations of Lep, sFlt-1, and PlGF were measured by quantitative sandwich ELISA using speciesspecific commercial kits from R\&D System Inc. (Minneapolis, MN).

\section{Liquid chromatography-tandem mass spectrometric (LC/MS/MS) analysis}

Serum concentrations of 16 Cers and 10 DHCers were measured by liquid chromatography-tandem mass spectrometric assay. The measurements were implemented for sera by the analytical methodology as previously described .

\section{Statistical analyses}

The differentiating power of each gene in non-PE and PE placental tissues from multiple GEO datasets was combined by meta-analysis. The optimal Cer marker in women's serum was determined by Mann-Whitney $\mathrm{U}$ test P-value, fold change, and AUC in the testing cohort. Performance of Lep, the optimal Cer marker, and Lep/Cer ratio in predicting PE was measured in the validation cohort by calculating AUC, sensitivity, specificity, PPV, and NPV. A time-to-event analysis was performed to calculate the gap between the time of prediction and the time of confirmatory diagnosis of PE. Results were compared with a reference point sFlt-1/PlGF ratio. Statistical analyses were preformed using $\mathrm{R}$ packages .

\section{RESULTS}

Meta-analysis confirmed the significant elevation of placental Lep expression in PE placentas 
To test the ability of placental Lep levels to differentiate non-PE from PE pregnancies, we performed a meta-analysis of gene expression profiles based on six PE placental studies (GSE4707, GSE10588, GSE24129, GSE25906, GSE44711, and GSE54618; see Table I). Among the 13,137 genes, Lep in PE placentas had the maximal change $(1.9$-fold $)$ and the most significant difference $(P<0.0001)$ compared with non-PE placentas (Fig. 2 and Appendix 1). Furthermore, in each study, Lep levels were higher in PE compared with non-PE placentas $(P<0.05$; Fig. 3$)$. The overexpression of the Lep transcripts was found to be significant in all PE women (including early-onset, late-onset, and severe PE).

PubMed meta-analysis identified Cer as the downstream metabolic messenger of Lep cascade

To discover the potential metabolic messengers downstream of Lep signaling cascade, we conducted a literature mining study based on the PubMed database. With the keyword of "Leptin; Lipid Metabolism", 4212 publications were obtained from years of 2000 to 2020. Among 4212 publications, 78 studies were designed to investigate the biology underlying the cytoplasmic interactions between Lep and Cer at the molecular level. Afterwards, 14 and 150 publications were obtained by searching the keywords of "Ceramide; Pregnancy" and "Ceramide; Preeclampsia", respectively, suggesting potential mechanistic implications of Cer in PE pathophysiology. Based on these results, Cer and its biosynthetic precursor dihydroceramide (DHCer) were selected as the biomarker candidates to generate our hypothesis and launched the following analyses.

\section{Serological quantitative analysis confirmed Lep, Cer, and DHCer as maternal markers for PE at diagnosis}

We measured levels of circulating Lep in women from the testing cohort (testing cohort included 7 non-PE and 8 PE women; demographic data are shown in Table II). Concentrations of Lep were significantly higher in $\mathrm{PE}$ women than in women without $\mathrm{PE}$ between 25 and 31 wks' of gestation $(P=0.02,2.97$-fold; Fig. 4A, Appendices 2 and 3).

We also characterized gestational profiles of 16 Cers and 10 DHCers in maternal sera (Fig. 4, Appendices 2 and 3). A total of 11 candidates (4 Cers and 7 DHCers) were significantly altered in PE $(P<0.05$; Fig. 4A). Among the 11 candidates, Cer (d18:1/25:0) was found to be the only Cer that significantly changed compared with Lep in PE women (0.88-fold).

\section{Lep/Cer (d18:1/25:0) ratio differentiated PE from normal pregnancies}

The Lep/Cer (d18:1/25:0) ratio had the largest area under the curve (AUC) compared with other marker combinations (Fig. 4B and Appendix 4) for differentiating PE from non-PE women in the testing cohort. Furthermore, compared with the individual Lep and Cer (d18:1/25:0) levels, the Lep/Cer (d18:1/25:0) ratio showed a lower $P$ (0.006 vs 0.02 and 0.03 , respectively), a larger fold change (4.04 vs 2.97 and 0.88 , respectively), and a higher AUC (0.911 vs 0.875 and 0.839, respectively; Table III and Appendix 5).

\section{Validation analysis found that Lep/Cer (d18:1/25:0) ratio predicts PE early in gestation}

When we evaluated the predictive performance of the Lep/Cer (d18:1/25:0) ratio in the validation cohort (20 women without PE with 55 samples, and 20 women with PE with 51 samples; see Table IV and Fig. 5), We found that among the $20 \mathrm{PE}$ women, 5 and 13 had early- and late-onset PE, respectively. The dates of diagnosis were missing in the remaining 2 women.

We observed an increase in serum Lep $(P<0.0001 ; 1.71$-fold $)$ and a decrease in Cer $(\mathrm{d} 18: 1 / 25: 0)(P<$ 0.0001 ; 0.70-fold) in PE women at 5 to 29 wks' of gestation (Appendix 6), which resulted in an elevated Lep/Cer (d18:1/25:0) ratio (Fig. 6). Most notably, this ratio was a significantly better predictor of all types of $\mathrm{PE}(\mathrm{AUC}=0.887)$ than Lep $(\mathrm{AUC}=0.809 ; P=0.0006)$ or Cer $(\mathrm{d} 18: 1 / 25: 0) \quad(\mathrm{AUC}=0.790$; $P=0.008)$ levels alone. In addition, the ratio performed well at wider gestational windows from 5 to 15 and 16 to 29 wks (AUC $=0.876$ and 0.892 , respectively) than the individual markers: Lep (AUC = $0.868 ; P=0.4$ and $0.824 ; P=0.1$, respectively) and Cer $(\mathrm{d} 18: 1 / 25: 0)(\mathrm{AUC}=0.868 ; P=0.1$ and 0.747 ; $P=0.02$, respectively). These results were consistent with the univariate and multivariate results found in non-obese women (Appendix 7). The Lep/Cer (d18:1/25:0) ratio accounted for $31 \%$ of the variation 
in the PE outcome, higher than that with Lep (15\%), Cer (d18:1/25:0) (15\%), and pre-pregnancy body mass index (BMI) (8\%). In a multivariate analysis, the explained variance of Lep was dominant early in gestation (5 to 15 wks), while Cer (d18:1/25:0) was dominant in mid-gestation (16 to 29 wks; Appendix 7B). The longitudinal profiling of Lep and Cer (d18:1/25:0) levels improved the predictive performance of Lep/Cer (d18:1/25:0) ratio. Moreover, the Lep/Cer (d18:1/25:0) ratio outperformed the sFlt-1/PlGF ratio in predicting impending PE, with a lower $P$ (Fig. 6) and a higher AUC $(P<0.0001$; Appendix 6D).

Time-to-event analysis at 5 to 25 wks (Fig. 7) compared the Lep/Cer (d18:1/25:0) ratio and the sFlt-1/PlGF ratio in predicting the impending $\mathrm{PE}$. Among the $18 \mathrm{PE}$ women with known diagnoses dates, $83 \%$ (15/18; 5 early-onset and 10 late-onset) were identified by the Lep/Cer (d18:1/25:0) ratio 11 or more weeks prior to their clinical diagnosis. In contrast, the sFlt-1/PlGF ratio only identified $22 \%(4 / 18 ; 1$ early-onset and 3 late-onset) of $\mathrm{PE}$ women 11 or more weeks prior to the diagnosis. The Lep/Cer (d18:1/25:0) ratio was able to predict impending PE a median of 23.0 [95\% confidence interval (CI): 12.8, 30.7] wks prior to the confirmatory diagnosis.

As shown in Fig. 8A, the Lep/Cer (d18:1/25:0) ratio correctly classified 85\% (17/20) of women with impending PE and $90 \%(18 / 20)$ of pregnancies without PE at 5 to 25 wks, giving a sensitivity of $85 \%(17 / 20)$, a specificity of $90 \%(18 / 20)$, a positive predictive value (PPV) of $89 \%(17 / 19)$, and a negative predictive value (NPV) of $86 \%(18 / 21)$. In contrast, $40 \%(8 / 20)$ of women with subsequent PE and $45 \%(9 / 20)$ of women without PE were correctly classified by the sFlt-1/PlGF ratio, yielding a sensitivity of only $40 \%$ (8/20), a specificity of $45 \%(9 / 20)$, a PPV of $42 \%$ (8/19), and a NPV of $43 \%(9 / 21)$ (Fig. 8B). In addition, the Lep/Cer (d18:1/25:0) ratio had a higher AUC than the sFlt-1/PlGF ratio at 5 to 25 wks [0.92 (95\% CI: $0.86,0.98)$ vs. $0.52(95 \%$ CI: $0.39,0.64) ; P<0.001]$.

\section{DISCUSSION}

Early prediction of PE remains a challenge in current clinical practice. Known traditional risk factors inadequately identify women who will develop PE early in gestation. To aim the discovery of novel serological markers with better predictive power for $\mathrm{PE}$ at early gestations, we applied a multi-omics approach, integrating differentially expressed gene (DEGs) from placental mRNA expression multiplex analysis and lipidomic database mining of existing literature, to identify novel PE biomarkers, which are Lep and Cer. By characterizing the maternal serological profiles of Lep and Cer using commercial ELISA and reported liquid chromatography-tandem mass spectrometry (LC/MS/MS) assays, we validated the up-regulated Lep and down-regulated Cer(d18:1/25:0) in a case-control testing cohort with maternal sera collected at confirmative diagnosis of PE. With a cohort of longitudinally collected maternal sera from both PE and non-PE women, we further assessed the PE-predictive power of the Lep/Cer (18:1/25:0) ratio early in gestation. Our results validated this ratio as a better serological predictor of impending PE than the established sFlt-1/PlGF ratio. Our findings demonstrated that the use of the Lep/Cer (d18:1/25:0) ratio can identify women at high risk of developing $\mathrm{PE}$ at a substantially earlier time window during pregnancy (at 5 to 25 wks) than the sFlt-1/PlGF ratio (after 25 wks). Moreover, our results showed that, compared with the sFlt-1/PlGF ratio, the Lep/Cer (d18:1/25:0) ratio has better sensitivity ( $40 \%$ vs $85 \%$ ), specificity ( $45 \%$ vs $90 \%$ ), PPV ( $42 \%$ vs $89 \%$ ), and NPV (43\% vs 86\%). Therefore, early in gestation, the Lep/Cer (d18:1/25:0) ratio outperforms the established sFlt-1/PlGF ratio and is a predictor of impending PE. The fact that the Lep/Cer (d18:1/25:0) ratio increases early in gestation in pregnant women who later develop PE offers an opportunity for predicting $\mathrm{PE}$ prior to the onset of clinical signs and symptoms. Integration of the ratio into a high-risk screening tool might allow patient identification at a pre-symptomatic stage. In addition, the concept of integrating a transcriptomic approach in placenta tissue with a lipidomic approach in serum is novel, as it combines the merits of studies in tissue whose focuses are more towards the pathogenesis and pathophysiology with those study in serum whose focuses are more towards the clinical translation. Taking the candidates obtained from the discovery phase to the validation phase makes the findings of this study translatable into clinical practice.

Previous studies have suggested that placental trophoblast cells are a leading source of circulating Lep in pregnancy, where Lep increases progressively in the first and second trimesters, peaks in the third, and 
returns to pre-pregnancy levels prior to parturition . In early gestation, Lep may play a critical role in modulating essential biological activities such as proliferation, protein synthesis, invasion, and apoptosis of trophoblast cells. Failure of trophoblastic invasion might result in incomplete remodeling of the maternal spiral arteries and inadequate placental perfusion to the embryo, leading to various disorders of reproduction and gestation such as intrauterine growth restriction, $\mathrm{PE}$, gestational diabetes mellitus, and recurrent miscarriage. Other recent studies documented significant elevations of Lep expression in preeclamptic placentas . In the current study, we found a significant upregulation of Lep in maternal sera of PE women, which is consistent with previous reports .

The gestational dysregulation of Cer metabolism is believed to induce the aberrant de novo synthesis and lysosomal breakdown of Cer, which leads to trophoblast cell autophagy, dysfunctional development of placenta, and eventually pregnancy complications like PE . Cer (d18:1/25:0) is an unusual odd-chain species of the Cer family that is generated by de novo synthesis based on 25:0 fatty acid. Such odd-chain fatty acids are mainly from dairy products and meat from ruminant animals . Cer (d18:1/25:0) was previously described as a potential urinary marker of inflammation-induced alcoholic liver disease . Recently, an elevation of the Cer (d18:1/25:0) was also identified as a serum prognostic marker to predict various acute diseases, including cardiovascular death, myocardial infarction, and stroke in patients with acute myocardial infarction within an ensuing 12-month period. Our results suggested the pathological implications of Cer (d18:1/25:0) in the development of pregnancy complicated by $\mathrm{PE}$, which might provide additional insights into the mechanistic roles of Cer (d18:1/25:0) and other odd-chain Cer species in PE pathophysiology.

An association between Lep and Cer has been reported in several studies. Lep was shown to exert its anorexigenic action by promoting mitochondrial lipid oxidation in both adipose and non-adipose tissues to alleviate ectopic accumulation of lipotoxic Cer via the activation of AMP-activated protein kinase (AMPKK/AMPK) . The de novo synthesis of Cer was found to play prominent roles in modulating downstream signaling of central Lep's activity via mediation of malonyl-CoA, carnitine palmitoyl transferase-1c, and serine palmitoyl transferase. Persistent elevation of circulating Lep also appears to induce resistance at the level of the Lep receptor, which accounts for attenuated potency of Lep to alleviate the accumulated cytotoxic Cer . Our data suggest a crosstalk between Lep's activity and de novo Cer synthesis. Lep functions well as a predictor of PE early in gestation, while Cer (d18:1/25:0) performs better at mid-gestation. The Lep/Cer (d18:1/25:0) ratio has a better predictive performance than Lep or Cer (d18:1/25:0) levels alone. Our findings revealed a correlation between the biological patterns of the two markers during PE progression, which might add value to existing knowledge about the Lep-Cer relationships.

Our study has several limitations. First, the sample sizes of the cohorts were small and lacked racial heterogeneity-thus, the generalizability of the results awaits larger and more racially diverse study populations. Second, longitudinal collections of blood samples were not evenly distributed over gestation. Finally, we did not investigate the exact tissue of origin, where Lep is overexpressed in PE women. By using a conditional knock-in placental Lep transgenic mouse model, it may be possible to elucidate the mechanistic role of placental Lep in the pathogenesis of PE early in pregnancy.

\section{CONCLUSIONS}

The disruptions of gestational homeostasis involving placenta-related biological networks are important factors contributing to the pathophysiology of PE. Lep, an endocrine regulator of body energy repletion, and Cer (d18:1/25:0), a bioactive metabolic messenger downstream of Lep, were identified by the multi-omics discovery to be significantly up- and down-regulated in the maternal circulation of women with PE. The Lep/Cer (d18:1/25:0) ratio was demonstrated to provide augmented predictive power in differentiating $\mathrm{PE}$ from a pregnancy without PE before a confirmatory diagnosis can be made. The maternal Lep/Cer (d18:1/25:0) ratio, with an earlier elevation in gestation, is a superior prognostic marker than the sFlt-1/PlGF ratio. If validated as a laboratory developed test or in vitro diagnostics, the deployment of the Lep/Cer ratio test to assess $\mathrm{PE}$ and proactively manage asymptomatic early pregnancies should have profound impact on $\mathrm{PE}$ care. 


\section{Acknowledgements}

We thank our colleagues in the March of the Dimes Prematurity Research Center at Stanford University and Pediatrics Proteomics Group for critical discussions.

Author contributions : XBL, KGS, and HJC contributed to concept development and design.

JY, RJW, and DKS contributed to the acquisition of data.

QH, SH, XY, JY, ZL, JS, ZL, ST, WL, XZ, LM, SL, RJW, GMS, DKS, JCW, and DBM contributed to the analysis and interpretation of data.

$\mathrm{QH}$ and $\mathrm{SH}$ drafted the manuscript.

XY, JY, ZL, JS, ZL, ST, WL, XZ, LM, SL, RJW, GMS, DKS, HJC, JCW, DBM, KGS, and XBL critically revised the manuscript.

All the authors gave final approval of the version to be submitted and agreed to be accountable for all aspects of the work.

Data and materials availability : The datasets used and/or analyzed in this study are available upon request to the corresponding author.

\section{Conflict of interest}

The authors declare that they have no conflicts of interest.

\section{Funding}

None.

\section{Reference}

\section{Hosted file}

2021.01.11.Figure.pdf available at https://authorea.com/users/390542/articles/504850case-finding-of-early-pregnancies-at-risk-of-preeclampsia-using-maternal-blood-leptinceramide-ratio-multi-omics-discovery-and-validation-from-a-longitudinal-study

\section{Hosted file}

2021.01.11.Table.pdf available at https://authorea.com/users/390542/articles/504850-casefinding-of-early-pregnancies-at-risk-of-preeclampsia-using-maternal-blood-leptinceramide-ratio-multi-omics-discovery-and-validation-from-a-longitudinal-study 